\title{
论 文
}

\section{基于着陆器地基测量的火星定向参数确定}

\section{杨轩 ${ }^{1,2}$, 跒建国 ${ }^{1 *}$, Le Maistre Sebastien ${ }^{2}$, Dehant Véronique ${ }^{2}$, 叶茂 ${ }^{1}$, 金炜桐 ${ }^{1}$, 李斐 ${ }^{1}$}

1. 武汉大学测绘遥感信息工程国家重点实验室, 武汉 430079;

2. Royal Observatory of Belgium, Brussels B1180, Belgium

*联系人, E-mail: jgyan@whu.edu.cn

收稿日期: 2020-01-13; 接受日期: 2020-04-23; 网络出版日期: 2020-10-16

国家自然科学基金(编号：U1831132，41374024)、国家自然科学基金青年科学基金(编号：41804025)、湖北省自然基金重点项目(编号: 2015CFA011, 2018CFA087)、澳门科技大学月球与行星科学实验室开放课题(编号: FDCT 119/2017/A3)和国家留学基金(CSC)资助

摘要 火星定向参数的精确测定对约束火星内部结构以及极区干冰变化的建模具有重要意义. 基于未来的火星 着陆任务, 仿真分析了利用双程多普勒与距离测量方法直接跟踪着陆器, 解算火星定向参数所能达到的精度. 结 果表明, 通过双程多普勒和双程测距直接跟踪火星着陆器超过 200 个工作日, 将显著提高火星岁差、章动以及日 长变化参数的精度, 约 800 个工作日后, 岁差参数精度可较目前提高5-10倍, 章动参数精度可达到10-30 mas, 日长 变化与钱德勒摆动参数精度可收玫至 5-10 mas, 这一精度水平可以满足研究火星内部结构与大气物质交换的需 要. 通过分析不同纬度着陆器的解算结果, 发现高纬度的着陆器有必要进行距离测量, 以保持岁差和章动参数的 解算精度. 此外, 研究还发现, 当火星星历误差小于 $75 \mathrm{~m}$ 时, 双程多普勒数据解算火星定向参数的精度不受影响, 而对于双程测距数据来说, 只有当火星历表精度优于 $0.15 \mathrm{~m}$ 时, 定向参数的解算结果才具有可信性.

关键词 火星着陆器, 火星定向参数, 多普勒, 距离测量

PACS: 96.30.Gc, 95.55.Pe, 91.10.Fc

\section{1 引言}

由于着陆器固定在行星表面，与行星自转联系紧 密, 对着陆器直接实施跟踪测量, 可精确测得行星的 定向参数信息 ${ }^{[1]}$. 定向参数包括岁差、章动、日长变 化(Length of Day, LOD)与极移等, 这些参数又与行星 的内部结构、内核大小与状态、行星表面质量分布等 息息相关 ${ }^{[2,3]}$. 通过对着陆器的跟踪测量, 不仅可满足 工程任务定位的需要，也可加深对行星内部构造、表
面质量转移的认识, 进一步深化深空探测的科学产出.

对火星着陆器测量包括地基(Direct-to-Earth, DTE)与星基(Lander-Orbiter-Link, LOL)两种方式. 地 基即地面深空站直接对着陆器跟踪测量, 获取行星着 陆器至地球深空站之间的链路信息 ${ }^{[1]}$; 而星基则是由 行星轨道环绕器与着陆器之间进行通信, 获得的是环 绕器与着陆器之间的链路信息 ${ }^{[4]}$. 两种方式各有优劣, 地基测量由于直接对火星着陆器实施跟踪, 避免了环 绕器轨道误差的影响, 操作起来更简便. 同时由于火

引用格式: 杨轩, 征建国, Le Maistre Sebastien, 等. 基于着陆器地基测量的火星定向参数确定. 中国科学: 物理学 力学 天文学, 2021, 51: 029501 Yang X, Yan J-G, Le Maistre S, et al. Mars orientation parameters determination based on direct-to-Earth measurement (in Chinese). Sci Sin-Phys Mech Astron, 2021, 51: 029501, doi: 10.1360/SSPMA-2020-0005 
星着陆器一般位于赤道附近, 环绕器一般为极轨卫星, DTE多普勒测量值对岁差、章动与日长变化参数更敏 感, LOL模式则更易于极移参数的解算 ${ }^{[4,5]}$.

着陆器跟踪数据解算火星定向参数最早可追溯到 20 世纪70年代. 海盗1号和 2 号(Viking)着陆器跟踪数据 首次应用于火星定向参数测定中 ${ }^{[6,7]}$. 随后, 文献[8]使 用火星探路者号(Mars Pathfinder)与海盗系列着陆器 的射电跟踪数据, 精化了火星定向参数, 同时约束了 火星内部结构, 结果暗示火星存在一个致密的核. 由 于2009年勇气号(Spirit)车轮陷入软土, 无法动弹, 2012年机遇号(Opportunity)为了保持能源供应在火星 表面停止运动超过 4 个月, 这期间的跟踪数据也为火星 定向参数的解算提供了新的数据源 ${ }^{[9,10]}$. 已于 2018 年 11 月成功着陆于埃律西昂平原(Elysium Planitia)的洞察 号(InSight)以及将要发射的ExoMars 2022探测器预期 将会极大地提高火星定向参数的精度 ${ }^{[2,11]}$. 其中ExoMars 2022着陆器将携带LaRa(Lander Radioscience)转 发器, 由比利时皇家天文台(Royal Observatory of Belgium, ROB)研制设计, 实施DTE多普勒测量. 同时新一 代LaRa(LaRa update)也已经在规划中, 可同时进行 DTE多普勒和距离测量. 我国计划于2020年首次发射 火星探测器, 包括一个环绕器与火星车, 任务期间将 对火星车的定位进行相关实验; 同时, 下一次火星探 测也正在规划当中.

本文以我国2020年火星车的着陆候选地为背景, 分析了使用DTE解算火星定向参数的方法以及所能达 到的精度. 此外, 还考虑了不同纬度的着陆器对解算精 度的影响. 文章第 1 节介绍了着陆器跟踪数据解算火星 定向参数的历史; 第2节简要介绍了目前火星固联坐标 系到惯性系之间的转换方法; 第3节分析了火星定向参 数对距离与多普勒观测值的影响; 第4节以目前大多数 着陆器纬度为基础, 仿真模拟了着陆器测量解算火星 定向参数的精度; 第 5 节把结果推广到全球，仿真相同 条件下, 着陆器纬度的变化与定向参数解算精度相互 关系; 第6节分析了火星星历误差对定向参数解算精 度的影响; 最后分析仿真结果, 总结全文.

\section{2 火星定向参数与坐标系统}

火星与地球拥有相似的自转倾角 (约 $\left.25.19^{\circ}\right)$ 与自 转周期(24 h 37 min $22.6 \mathrm{~s}$ ), 也存在岁差、章动和极移
现象. 在太阳和其他大天体的引力作用下, 火星自转轴 绕着黄道面的垂直轴旋转的长周期运动, 形成岁差; 在 空间绕着黄道轴转动的同时，由于潮汐力、内核等影 响, 还伴随有许多短周期的微小变化, 这些微小的角 即是章动角. 此外, 内核运动以及表面质量季节性等 变化导致火星日长变化与极移. 这些参数可统称为火 星定向参数(Mars Orientation Parameters, MOP), 可用 于火星固联坐标系到惯性坐标系之间的转换，其转换 关系可表示为 ${ }^{[12,13]}$ :

$\boldsymbol{r}_{b f}=\boldsymbol{R}_{y}\left(-X_{\mathrm{p}}\right) \boldsymbol{R}_{x}\left(-Y_{\mathrm{p}}\right) \boldsymbol{R}_{z}(\phi) \boldsymbol{R}_{x}(I) \boldsymbol{R}_{z}(\psi) \boldsymbol{R}_{x}(J) \boldsymbol{R}_{z}(N) \boldsymbol{r}_{i c f f}$,

其中, $\boldsymbol{r}_{b f}$ 为火星固联参考系下的位置矢量, $\boldsymbol{r}_{i c r f}$ 为相应 的位置矢量在天球坐标系下的表示, $X_{\mathrm{p}}$ 与 $Y_{\mathrm{p}}$ 表示极移, $\phi$ 为火星自转角, $I$ 和 $\psi$ 表示火星岁差章动角. 角 $J$ 和 $N$ 是 常量, $N$ 表示地球春分点至火星 $\mathrm{J} 2000$ 平赤道与地球 $\mathrm{J} 2000$ 平赤道交点的角度, 而 $J$ 表示火星 $\mathrm{J} 2000$ 平赤道与 地球J 2000 平赤道面之间的夹角, $\boldsymbol{R}_{x}, \boldsymbol{R}_{y}, \boldsymbol{R}_{z}$ 为3维旋转 矩阵. 这些旋转角可进一步表示为

$I=I_{0}+\dot{I}_{0} t+I_{\text {nut }}$,

$\psi=\psi_{0}+\dot{\psi}_{0} t+\psi_{\text {nut }}$,

$\phi=\phi_{0}+\dot{\phi}_{0} t+\delta \phi-\psi_{\text {nut }} \cos (\varepsilon)$.

火星岁差章动角 $I$ 与 $\psi$ 使用一系列参数的线性组 合来表示, 分别为 $\mathrm{J} 2000$ 时刻的常数项、岁差改正项与 章动改正项. 章动项使用一系列三角函数的线性组合 表示. 自转角 $\phi$ 包括常数项、自转速率、日长变化以及 岁差章动修正项，这一修正项主要是把自转角的参考 点修正到火星平赤道面与火星平轨道面的交点, 因此 在日长变化参数 $\delta \phi$ 中不含章动项的影响 ${ }^{[12]}$.

火星极移是由大气与表面质量交换等原因导致自 转角动量变化引起的, 因此极移呈现季节性变化, 与火 星公转周期成比例. 除此之外, 与地球类似, 火星也存 在钱德勒摆动(Chandler Wobble, CW) 的现象, 周期约 为 $205 \mathrm{~d}^{[13,14]}$. 因此极移参数可表示为

$X_{\mathrm{p}}=\sum_{m=1}^{5}\left(X_{c m} \cos \left(2 \pi f_{m} t\right)+X_{s m} \sin \left(2 \pi f_{m} t\right)\right)$,

$Y_{\mathrm{p}}=\sum_{m=1}^{5}\left(Y_{c m} \cos \left(2 \pi f_{m} t\right)+Y_{s m} \sin \left(2 \pi f_{m} t\right)\right)$,

其中, 前四项 $f_{m}=m / 687$ 表示由季节性导致的频率, 最 
后一项 $f_{5}$ 表示钱德勒摆动频率.

火星坐标系之间也可使用国际天文联合会(International Astronomical Union, IAU)推荐的方法, 采用火 星赤经、赤纬与本初子午线角来转换，相关转换矩阵 与角度可参考文献[15], 本文不再赘述, 两种转换方法 可得到相似的精度.

\section{DTE测量值与火星定向参数}

由于着陆器位于行星表面运行或固定不动，利用 多普勒或距离测量对着陆器进行定位不需要建立复杂 的动力学模型, 只需要运用行星历表和自转定向信息 就可确定着陆器位置, 此方法曾成功用于嫦娥三号着 陆器定位 ${ }^{[16,17]}$ 以及洞察号着陆后的快速定位. 双程测 量模式示意图如图1所示. 其中, 地球深空站在 $t_{1}$ 时刻 向火星发生上行信号, 着陆器在 $t_{2}$ 时刻接收信号同时 转发回地球, $t_{3}$ 时刻深空站接收信号并记录接收时间. 若地面发射站与接收站相同，则为双程测量，如果发 射站与接收站不同，则称为三程测量 ${ }^{[18,19]}$. 目前深空 探测一般使用 $\mathrm{S}$ 和X波段上行与下行频率，其距离与多 普勒测量值 $60 \mathrm{~s}$ 积分随机噪声可达 $1 \mathrm{~m}$ 与 $0.05 \mathrm{~mm} / \mathrm{s}$ 左 右 ${ }^{[1-3,13]}$. Ka波段具有比X波段更高的测量精度，已成 功应用于卡西尼(Cassini)深空探测任务. 同时, 目前正 在进行的水星探测任务(BepiColombo)也具有 $\mathrm{Ka}$ 波段 上行与下行载波转发能力, $1000 \mathrm{~s}$ 积分多普勒测量值 有望达到 $3 \mu \mathrm{m} / \mathrm{s}^{[20]}$, 高精度的测量也会极大地提高行 星定向参数的精度.

文献[21]以地球为原点，建立了火星定向参数在

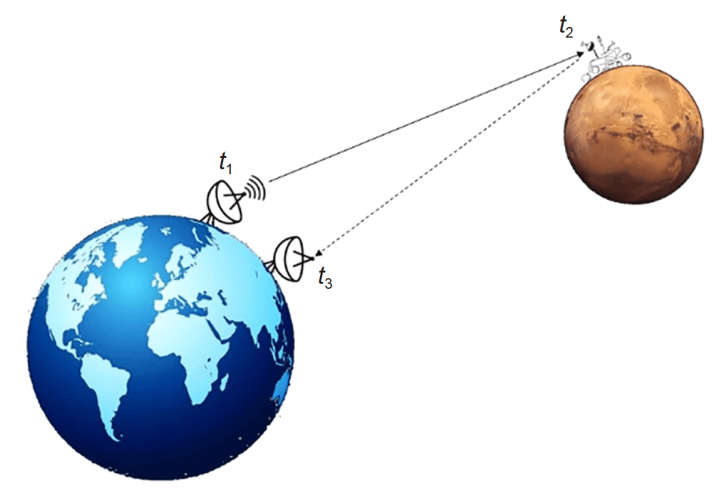

图 1 (网络版彩图)火星着陆器双程/三程测量模式示意图 Figure 1 (Color online) Two-way or three-way tracking mode for Mars lander.
距离观测值中的解析公式; 文献[22]给出了随着火星 自转轴位置的变化, 距离观测值的变化范围, 文献[13] 建立了随着着陆器的纬度变化, 多普勒观测值的变化 模型; 为简化公式, 文献[23]以火星为原点, 讨论了距 离和多普勒观测值中蕴含的火星定向参数信息. 下面 不经过推导, 直接给出距离和多普勒观测值火星定向 参数的一阶解析模型 ${ }^{[23]}$.

双程多普勒:

$$
\begin{aligned}
\Delta \dot{\rho}_{\dot{\psi}_{0}}= & \dot{\phi}_{0} R \dot{\psi}_{0} t \cos \theta\left(\cos \delta_{\mathrm{E}} \cos H_{\mathrm{E}} \cos I\right. \\
& \left.-\sin \delta_{\mathrm{E}} \sin \left(H_{\mathrm{E}}+\alpha_{\mathrm{E}}\right) \sin I\right), \\
\dot{\rho}_{\psi_{\text {nut }}}= & -\sin \delta_{\mathrm{E}} \dot{\phi}_{0} R \cos \theta \sin \left(H_{\mathrm{E}}+\alpha_{\mathrm{E}}\right) \psi_{\mathrm{nut}} \sin I, \\
\Delta \dot{\rho}_{I_{\text {nut }}}= & -\sin \delta_{\mathrm{E}} \dot{\phi}_{0} R \cos \theta \cos \left(H_{\mathrm{E}}+\alpha_{\mathrm{E}}\right) I_{\mathrm{nut}}, \\
\Delta \dot{\rho}_{\delta \phi}= & \dot{\phi}_{0} R \cos \theta \cos H_{\mathrm{E}} \cos \delta_{\mathrm{E}} \delta \phi, \\
\Delta \dot{\rho}_{X_{\mathrm{p}}}= & -\dot{\phi} R \sin \theta \cos \delta_{\mathrm{E}} X_{\mathrm{p}} \sin \left(\alpha_{\mathrm{E}}-\phi\right), \\
\Delta \dot{\rho}_{Y_{\mathrm{p}}}= & \dot{\phi} R \sin \theta \cos \delta_{\mathrm{E}} Y_{\mathrm{p}} \cos \left(\alpha_{\mathrm{E}}-\phi\right) .
\end{aligned}
$$

\section{双程测距:}

$$
\begin{aligned}
\Delta \rho_{\dot{\psi}_{0}}= & R \dot{\psi}_{0} t\left(\cos \delta_{\mathrm{E}} \sin H_{\mathrm{E}} \cos \theta \cos I-\cos \delta_{\mathrm{E}} \cos \alpha_{\mathrm{E}} \sin \theta \sin I\right. \\
& \left.+\sin \delta_{\mathrm{E}} \cos \theta \cos \left(H_{\mathrm{E}}+\alpha_{\mathrm{E}}\right) \sin I\right), \\
\Delta \rho_{\psi_{\text {nut }}}= & R \psi_{\text {nut }} \sin I\left(-\cos \delta_{\mathrm{E}} \cos \alpha_{\mathrm{E}} \sin \theta\right. \\
& \left.+\sin \delta_{\mathrm{E}} \cos \theta \cos \left(H_{\mathrm{E}}+\alpha_{\mathrm{E}}\right)\right),
\end{aligned}
$$

$\Delta \rho_{I_{\text {nut }}}=R I_{\text {nut }}\left(\cos \delta_{\mathrm{E}} \sin \alpha_{\mathrm{E}} \sin \theta-\sin \delta_{\mathrm{E}} \cos \theta \sin \left(H_{\mathrm{E}}+\alpha_{\mathrm{E}}\right)\right)$,

$\Delta \rho_{\delta \phi}=R \delta \phi \cos \theta \sin H_{\mathrm{E}} \cos \delta_{\mathrm{E}}$,

$$
\Delta \rho_{X_{\mathrm{p}}}=R X_{\mathrm{p}}\left(-\sin \delta_{\mathrm{E}} \cos \theta \cos \lambda+\cos \delta_{\mathrm{E}} \sin \theta \cos \left(\alpha_{\mathrm{E}}-\phi\right)\right),
$$

$\Delta \rho_{Y_{\mathrm{p}}}=R Y_{\mathrm{p}}\left(\sin \delta_{\mathrm{E}} \cos \theta \sin \lambda-\cos \delta_{\mathrm{E}} \sin \theta \sin \left(\alpha_{\mathrm{E}}-\phi\right)\right)$,

其中, $R$ 为火星平均半径, $\phi$ 为火星自转角, $I$ 和 $\psi$ 表示火 星岁差章动角, $\delta_{\mathrm{E}}$ 表示地球相对于火星的赤纬, $\theta, \lambda$ 为 着陆器纬度与经度, $\varepsilon$ 为火星自转轴倾角, $H_{\mathrm{E}}$ 为火星着 陆器位置的地球时角, $\alpha_{\mathrm{E}}$ 表示地球相对于火星的赤经.

从式(7)-(12)可以发现, 章动参数信息在双程多普 勒观测数据中, 与火星着陆器纬度 $\theta$ 、地球赤纬 $\delta_{\mathrm{E}}$ 的大 小高度相关. 当着陆器位于赤道时, 由章动引起的多普 勒观测值变化达到最大; 而当着陆器位于两极时, 多普 
勒观测值中将不含有任何章动信息. 着陆器当地时间 的地球时角 $H_{\mathrm{E}}$ 与地球相对于火星的赤经 $\alpha_{\mathrm{E}}$ 导致了多 普勒观测值中章动信息的周日项变化，并且两个章动 角所致的最大多普勒观测值出现在每天的不同时刻, 相距约 $6 \mathrm{~h}^{[23]}$. 地球赤纬 $\delta_{\mathrm{E}}$ 即火星-地球的连线与火星 赤道面的夹角，变化范围可达 $-25^{\circ}-25^{\circ}$, 绝对值越大 的 $\delta_{\mathrm{E}}$ 角越有利于章动参数解算, 但是对于日长变化参 数 $\delta \phi$, 地球赤纬 $\delta_{\mathrm{E}}$ 接近 $0^{\circ}$ 更适合参数求解; 由于式(7) 的首项占主导地位，因此近赤道着陆器与较小的地球 赤纬 $\delta_{\mathrm{E}}$ 适合岁差参数的求解; 而对于极移参数, 位于 赤道的着陆器将在多普勒观测值中不含任何极移信 息, 只有当着陆器位于两极时, 多普勒观测值中的极移 信息才能达到最大值.

相比较双程多普勒观测值，定向参数信息在双程 距离观测值中的表现有些许差异. 对比式(7)与(13)可 发现, 岁差在双程距离观测值中多了第二项, 此项量 级较小，式(13)的第一项和第三项与式(7)中多普勒观 测值峰值出现的时间不一致. 与多普勒中的章动和极 移信息不同，双程测距公式由于含有两项，因此受着 陆器纬度 $\theta$ 的影响较小。除周日项最大值出现的时间 不一致之外，双程距离观测值中的日长变化与多普勒 中的变化一致. 由于目前火星历表误差的存在，双程 距离观测值很少被用来解算火星定向参数 ${ }^{[24]}$. 随着越 来越多的探测器着陆在火星表面, 我们有理由相信, 未来的火星历表精度可得到极大的提升.

\section{DTE仿真模拟}

以往大多数成功的火星着陆器均位于赤道附近, 如海盗 1 号、火星探路者号、洞察号等, 未来将要进行 的美国Mars2020、欧洲航天局(European Space Agency)ExoMars以及我国首次火星任务都将着陆在 北纬 $20^{\circ}$ 附近, 因此我们以 $20^{\circ} \mathrm{N}$ 作为仿真中着陆器的纬 度. 式(7)-(16)显示, 定向参数信息在观测值中与着陆 器经度没有直接关系，经度的变化只会影响定向参数 信息的周日峰值出现的时间，每天不同跟踪着陆器的 时段会获得不同的参数精度水平. 仿真过程中利用式 (7)-(18), 合理安排着陆器的跟踪时段, 挑选定向参数 信息的周日项峰值时刻, 考虑到着陆器能源的限制, 每 天跟踪时间设置在 2-3 h, 同时考虑太阳辐射对测量噪
声的影响, 剔除太阳-地球-探测器(SEP)夹角小于 $15^{\circ}$ 的 情况, 图2为SEP与地球赤纬的变化.

\section{1 仿真模型}

仿真中使用Pathfinder火星定向参数模型，章动与 日长变化参数表示如下 ${ }^{[13]}$ :

$\psi_{\text {nut }}=\sum_{m=1}^{9} \psi_{m}^{\prime} \sin \left(a_{m} t+\theta_{m}\right)$,

$I_{\text {nut }}=\sum_{m=1}^{9} I_{m}^{\prime} \cos \left(a_{m} t+\theta_{m}\right)$,

$\delta \phi=\sum_{j=1}^{4} \phi_{c j} \cos \left(j l^{\prime}\right)+\phi_{s j} \sin \left(j l^{\prime}\right)+\sum_{j=1}^{3} \phi_{r j} \sin \left(j l^{\prime}\right)$,

其中, $\phi_{r j}$ 为相对论效应引起的日长变化改正项, $\phi_{c j}$ 与 $\phi_{s j}$ 为季节性的日长变化系数, $\psi_{m}^{\prime}$ 和 $I_{m}^{\prime}$ 可进一步表示为

$\psi_{m}^{\prime}=\psi_{m}\left(1+F \frac{\sigma_{m}^{2}}{\sigma_{m}^{2}-\sigma_{0}^{2}}\right)+\frac{I_{m}}{\sin I_{0}} F \frac{\sigma_{m} \sigma_{0}}{\sigma_{m}^{2}-\sigma_{0}^{2}}$,

$I_{m}^{\prime}=I_{m}\left(1+F \frac{\sigma_{m}^{2}}{\sigma_{m}^{2}-\sigma_{0}^{2}}\right)+\sin I_{0} \psi_{m} F \frac{\sigma_{m} \sigma_{0}}{\sigma_{m}^{2}-\sigma_{0}^{2}}$,

其中, $\psi_{m}$ 与 $I_{m}$ 为火星刚体章动振幅, $\sigma_{0}$ 为自由核章动参 数, $F$ 为内核参数. 仿真中设置为 $F=0.07, \sigma_{0}=-1.5 \% \mathrm{~d}$, 即 $\mathrm{FCN}=-240 \mathrm{~d}^{[13]}$. 着陆器位置设定在 $20^{\circ} \mathrm{N}, 40^{\circ} \mathrm{W}$, 待 估参数设置见表 1 .

一共包括 48 个待估参数，各参数之间相关性较强, 需要添加必要的先验约束. 章动参数的先验约束值设 置为非刚体章动(式(22)和(23), $\mathrm{FCN}=-230, F=0.12$ )与 刚体章动振幅差值的两倍; 自转变化参数的先验约束

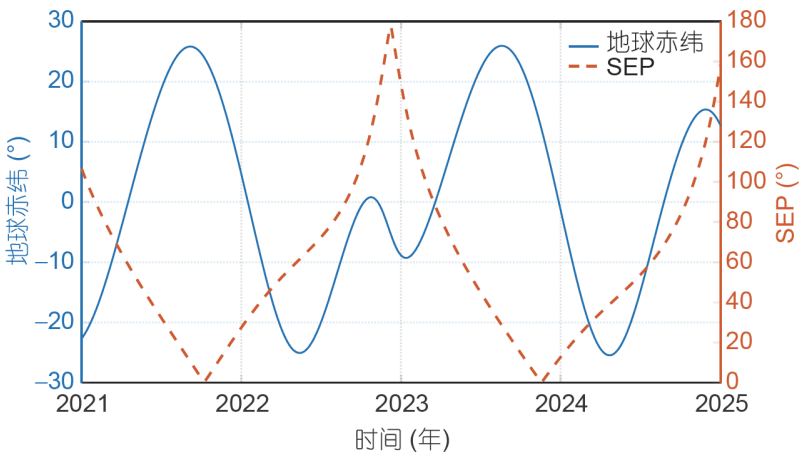

图 2 (网络版彩图)地球赤纬与太阳-地球-探测器夹角变化 Figure 2 (Color online) Earth declination and SEP variations versus time. 
表 1 待估参数设置

Table 1 Simulation settings

\begin{tabular}{|c|c|c|}
\hline 符号 & 数值 & 先验约束 \\
\hline$X(\mathrm{~km})$ & 2444.742 & 10 \\
\hline$Y(\mathrm{~km})$ & -2051.382 & 10 \\
\hline$Z(\mathrm{~km})$ & 1161.568 & 10 \\
\hline$\dot{\psi}_{0}(\mathrm{mas} / \mathrm{yr})$ & -7610.1 & 无 \\
\hline$I_{0}(\mathrm{mas} / \mathrm{yr})$ & -3.4 & 无 \\
\hline$\dot{\phi}_{0}($ degree/d $)$ & 350.891985307 & 无 \\
\hline$\phi_{c 1}, \phi_{s 1}$ (mas) & $481,-155$ & 20,24 \\
\hline$\phi_{c 2}, \phi_{s 2}(\mathrm{mas})$ & $-103,-93$ & 18,16 \\
\hline$\phi_{c 3}, \phi_{s 3}$ (mas) & $-35,-3$ & 16,14 \\
\hline$\phi_{c 4}, \phi_{s 4}$ (mas) & $-10,-8$ & 12,12 \\
\hline$I_{10}, \psi_{10}(\mathrm{mas})$ & $-0.4,-632.6$ & 24,19 \\
\hline$I_{20}, \psi_{20}(\mathrm{mas})$ & $0,-44.2$ & 5,8 \\
\hline$I_{30}, \psi_{30}(\mathrm{mas})$ & $0,-4.0$ & 40,96 \\
\hline$I_{40}, \psi_{40}(\mathrm{mas})$ & $-49.1,-104.5$ & 3,7 \\
\hline$I_{50}, \psi_{50}(\mathrm{mas})$ & $515.7,1097.0$ & 35,139 \\
\hline$I_{60}, \psi_{60}(\mathrm{mas})$ & $112.8,240.1$ & 268,569 \\
\hline$I_{70}, \psi_{70}(\mathrm{mas})$ & $19.2,40.9$ & $3,3.9$ \\
\hline$I_{80}, \psi_{80}(\mathrm{mas})$ & $3.0,6.5$ & $0.5,0.85$ \\
\hline$I_{90}, \psi_{90}(\mathrm{mas})$ & $0.4,1.0$ & $0.06,0.17$ \\
\hline$X_{c 1}, X_{s 1}$ (mas) & 13,13 & 13,13 \\
\hline$X_{c 2}, X_{s 2}$ (mas) & 8,8 & 8,8 \\
\hline$X_{c 4}, X_{s 4}$ (mas) & 2,2 & 2,2 \\
\hline$X_{c 5}, X_{s 5}$ (mas) & 55,55 & 55,55 \\
\hline$Y_{c 1}, Y_{s 1}$ (mas) & 5,5 & 5,5 \\
\hline$Y_{c 2}, Y_{s 2}(\mathrm{mas})$ & 8,8 & 8,8 \\
\hline$Y_{c 4}, Y_{s 4}$ (mas) & 2,2 & 2,2 \\
\hline$Y_{c 5}, Y_{s 5}$ (mas) & 34,34 & 34,34 \\
\hline
\end{tabular}

值按照当前实际精度的两倍来设置. 目前还没有确切 的研究给出火星极移的振幅, 但已有研究表明, 火星 极移的量级在 $0-15$ mas之间, 钱德勒摆动量级约在 10 100 mas之间 ${ }^{[25,26]}$, 依据文献[23]的研究, 火星极移量级 设置见表 1 , 并且设置为 $100 \%$ 误差. 仿真过程中, 首先 使用上述参数的“真值”生成双程多普勒和距离观测 值，在观测值上按照当前深空地面站与转发器的噪声 水平添加相应的噪声 $(60 \mathrm{~s}$ 积分双程多普勒 $0.05 \mathrm{~mm} / \mathrm{s}$; 双程测距 $1 \mathrm{~m})$ 以及为待估参数添加偏差 $(1 / 2$ 先验约束) 后，依据此观测值使用带有先验约束的最小二乘法来
反演定向参数与着陆器位置, 与参数的“真值”做对比, 分析参数解算的精度. 参数解算初期, 受观测值较少等 因素的影响, 只解算着陆器位置, 当着陆器位置精度稳 定到米级后, 开始同时解算火星定向参数.

\section{2 仿真结果}

着陆器位置收玫结果见图3. 使用 $1 \mathrm{~h}$ 的跟踪数据 可使 $X, Y$ 坐标收玫到米级. 由于具有良好的 $Z$ 方向敏感 度, 双程测距数据相比双程多普勒, 在 $Z$ 方向上精度要 高一个量级以上，2 h后可达到十几米，而双程多普勒 数据由于对 $Z$ 方向精度不敏感, 仿真初期精度为上百

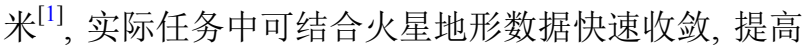
$Z$ 方向的精度 ${ }^{[24]}$. 图3中出现了 $Y$ 轴真实误差偏大的情 况，原因主要由于在前几个小时的跟踪数据解算中, 并没有对火星定向参数同时估计，而在仿真中，定向 参数根据目前参数精度人为地增加了偏差, 由此引入 了系统误差. 此外, 在图3中，约 $2 \mathrm{~h}$ 后出现收玫转折, 原因是仿真中设置每天观测 $2-3 \mathrm{~h}$, 由于跟踪数据的不 连续导致几何构型产生了迅速变化, 使得着陆器位置 收敛速度加快.

图4-7为火星定向参数的收玫过程. 其中, 在第200 任务日附近由于SEP夹角过小剔除部分数据, 产生了 间断. 经过约 200 任务日, 岁差参数 $\dot{I}_{0}, \dot{\psi}_{0}$ 的精度可超过 当前相应参数的精度. 随着观测数据的积累, 最终可提 高 $\dot{I}_{0}, \dot{\psi}_{0}$ 精度约5-10倍. 自转速率 $\dot{\phi}_{0}$ 则需要超过650任务 日才可超过当前精度，一方面是由于目前 $\dot{\phi}_{0}$ 的精度足 够高, 而另一方面, 以往处理着陆器数据并没有同时 估计章动与极移参数, 有可能导致 $\dot{\phi}_{0}$ 的精度过于乐观. 章动参数解算结果见图 5 , 为了使图片清晰易读, 只绘 制了精度变化范围较大的参数, 包括年周期项 $\left(I_{1}, \psi_{1}\right)$, 半年周期项 $\left(I_{5}, \psi_{5}\right)$ 以及 $1 / 3$ 年周期项 $\left(I_{3}, \psi_{3}, I_{6}, \psi_{6}\right)$. 其 中, $I_{6}$ 与 $\psi_{6}$ 精度的提升最为明显, 这是因为火星自由核 章动的频率与 $1 / 3$ 年周期接近, 发生的“共振”现象可增 加 $I_{6}$ 与 $\psi_{6}$ 的振幅范围. 最终, 章动可达到10-30 mas的精 度. 值得注意的是, 虽然需要近 200 任务日的观测数据 才可以超过目前参数的精度, 但是在实际情况下, 通过 加入已有的火星着陆器跟踪数据, 可以极大地提高参 数的收玫速度.

日长变化即火星自转轴惯性矩的变化，它反映着 火星极区干冰与大气之间的物质交换. 经过 700 任务 

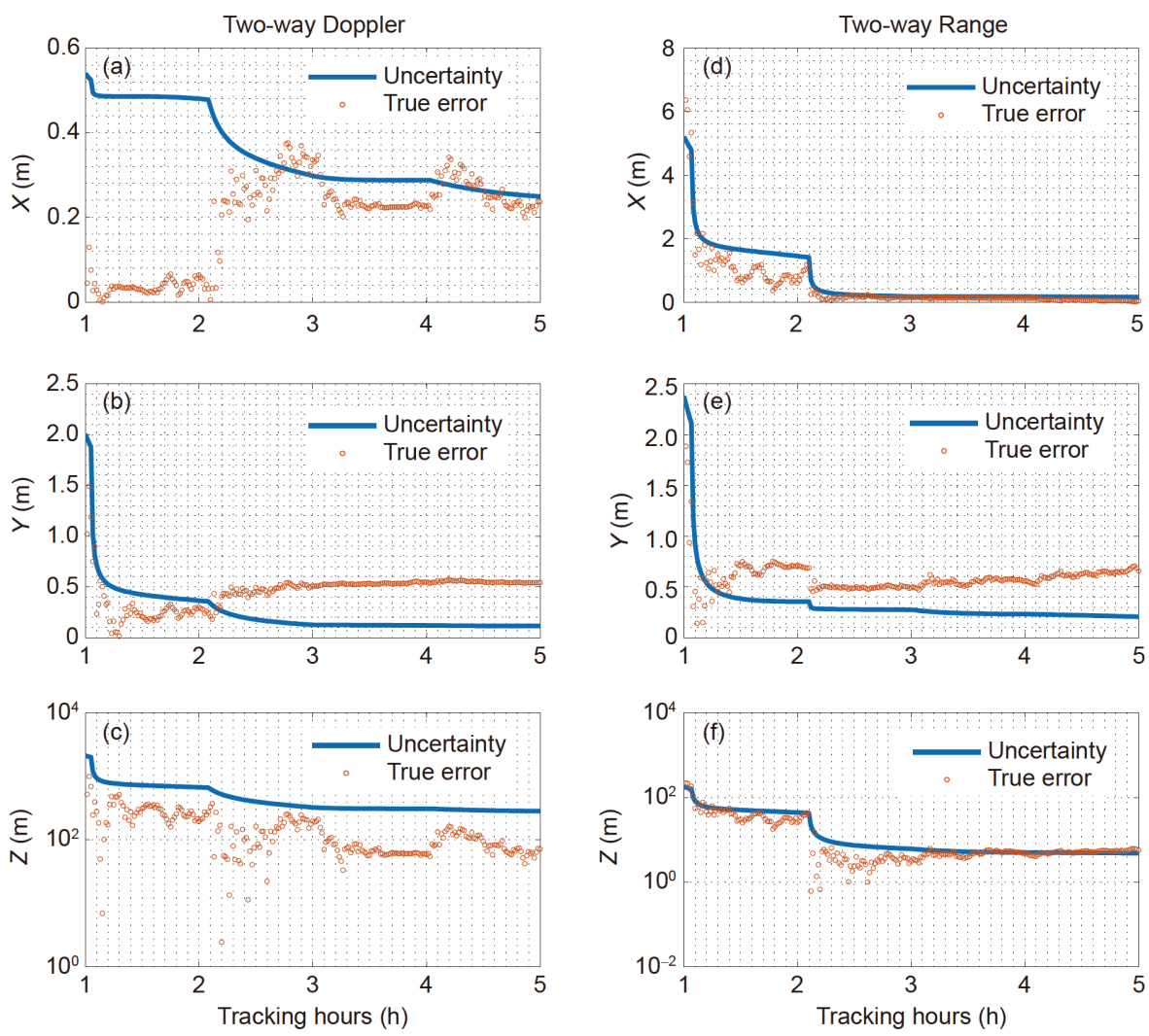

图 3 (网络版彩图)着陆器位置收玫曲线(实线为标准差, 红色圆圈表示与真值的差距, 即真实误差). 使用双程多普勒数据计 算着陆器位置 $X(\mathrm{a}), Y(\mathrm{~b}), Z$ (c)的收敛曲线; 使用双程测距数据计算着陆器位置 $X$ (d), $Y$ (e), $Z$ (f)的收敛曲线

Figure 3 (Color online) Lander positioning versus tracking duration (the solid line is the uncertainty of adjusted parameters and the red circle is the true error). The convergence curves for the lander positioning at $X(\mathrm{a}), Y(\mathrm{~b})$, and $Z$ (c) axes using two-way Doppler data; the convergence curves for the lander positioning at $X$ (d), $Y(\mathrm{e})$, and $Z$ (f) axes using two-way range data.

日, 日长变化参数的精度可小于 $5 \mathrm{mas}$, 满足大气之间 物质交换过程分析的需要 ${ }^{[24]}$. 关于极移，低纬度着陆 器位置 $\left(20^{\circ} \mathrm{N}\right)$ 制约着参数解算精度, 仿真结果显示只 有对预期振幅较大的钱德勒摆动项的精度有贡献，最 终精度优于 $5 \mathrm{mas}$, 可为火星黏弹性的研究提供重要的 约束.

综上, 着陆器位置和火星定向参数解算精度总结 见表2.

\section{5 不同着陆器纬度对解算结果的影响}

上述仿真中假设火星着陆器位于北纬 $20^{\circ}$, 根据式 (7)-(18), 不同纬度的火星着陆器会影响定向参数信息 在观测量中的量级大小, 进一步地, 火星定向参数的解 算精度会产生不同程度的改变. 为了量化精度变化的
幅度, 我们挑选出具有代表性的着陆器纬度, 重复上 述仿真计算, 最终定向参数的精度变化如图 8 所示.

如果只使用双程多普勒数据, 随着着陆器纬度的 增加, 火星岁差、章动和日长变化参数的解算精度将 随之降低, 而极移项(钱德勒摆动)的精度将会得到提 高. 位于赤道附近的着陆器仅使用双程多普勒数据观 测不利于极移的求解; 相反, 仅使用双程多普勒数据 跟踪位于两极附近的着陆器会为岁差、章动和日长变 化参数的求解带来麻烦. 增加双程测距数据后, 日长变 化参数精度仍然与着陆器纬度正相关, 岁差解算摆脱 了着陆器纬度的限制, 而章动参数与极移的精度变化 一致, 均为与着陆器纬度变化负相关. 综上, 高纬度的 着陆器只进行双程多普勒测量会显著降低岁差和章动 参数的解算精度, 因此有必要增加距离测量弥补多普 勒测量的不足. 

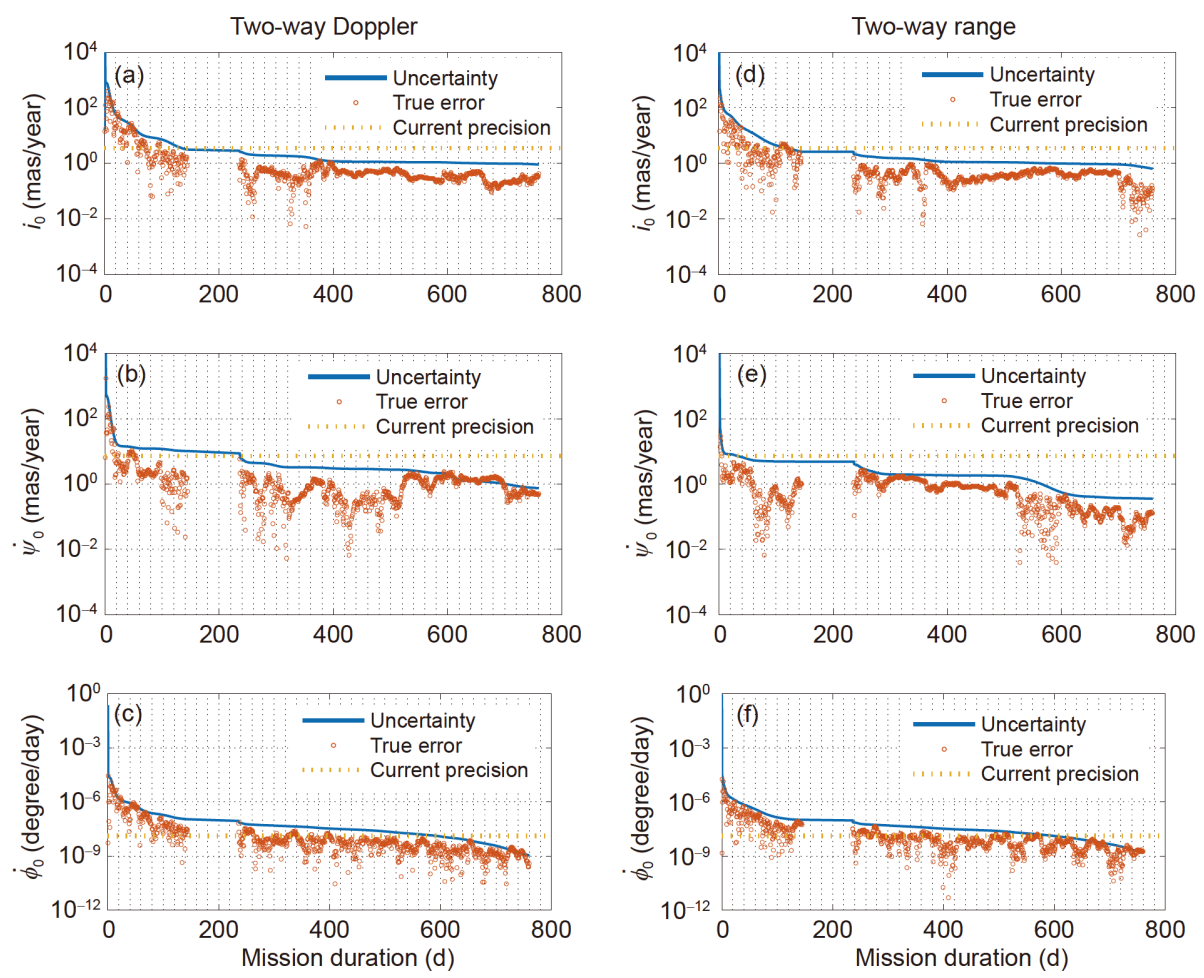

图 4 (网络版彩图)岁差与自转速率收玫曲线, 实线为标准差, 红色圆圈表示真实误差, 黄色虚线表示当前参数的精度, 数值来 源于已有的着陆器跟踪数据计算的结果 ${ }^{[13]}$. 使用双程多普勒数据计算岁差参数 $\dot{I}_{0}(\mathrm{a}), \dot{\psi}_{0}(\mathrm{~b})$ 和自转速率 $\dot{\phi}_{0}$ (c) 的收玫曲线; 使用 双程测距数据计算岁差参数 $I_{0}(\mathrm{~d}), \dot{\psi}_{0}(\mathrm{e})$ 和自转速率 $\dot{\phi}_{0}(\mathrm{f})$ 的收敛曲线

Figure 4 (Color online) Precession and spin rate parameters versus tracking duration. The solid line is the uncertainty of adjusted parameters and the red circle is the true error. The dashed line represents current precision of these parameters computed from lander tracking data. The convergence curves for the precession and rotation parameters $I_{0}(\mathrm{a}), \psi_{0}(\mathrm{~b}), \dot{\phi}_{0}$ (c) using two-way Doppler data. The convergence curves for the precession and rotation parameters $I_{0}(\mathrm{~d}), \dot{\psi}_{0}(\mathrm{e}), \dot{\phi}_{0}$ (f) using two-way range data.
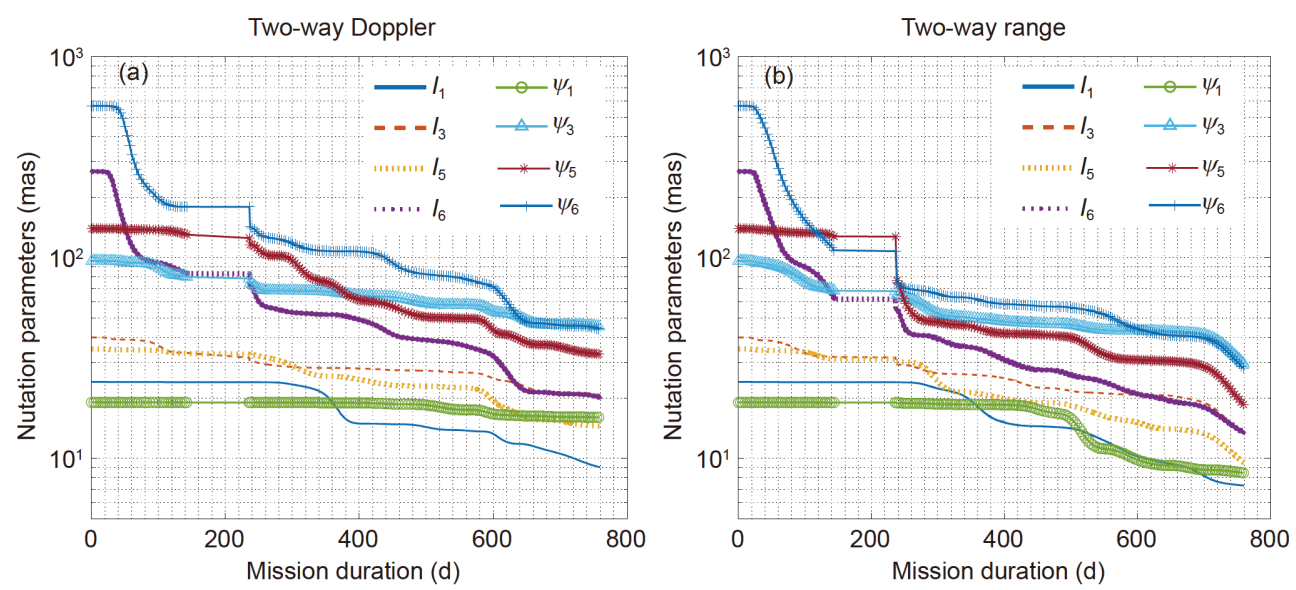

图 5 (网络版彩图)章动参数收玫曲线(为防止混淆, 图中的曲线全部为相应参数的标准差, 未绘出精度提升不大的章动参数, 图中所有参数的真实误差均小于两倍标准差). (a) 使用双程多普勒数据计算章动参数的结果; (b) 双程测距数据的计算结果

Figure 5 (Color online) Nutation parameters versus tracking duration. All lines in the figure are uncertainties of adjusting parameters. For avoiding confusion, the nutation parameters with little improvement in accuracy are not drawn. The true errors of all parameters are less than $2 \sigma$. (a) The result of using two-way Doppler data to determine nutation parameters; (b) the result of using two-way range data. 

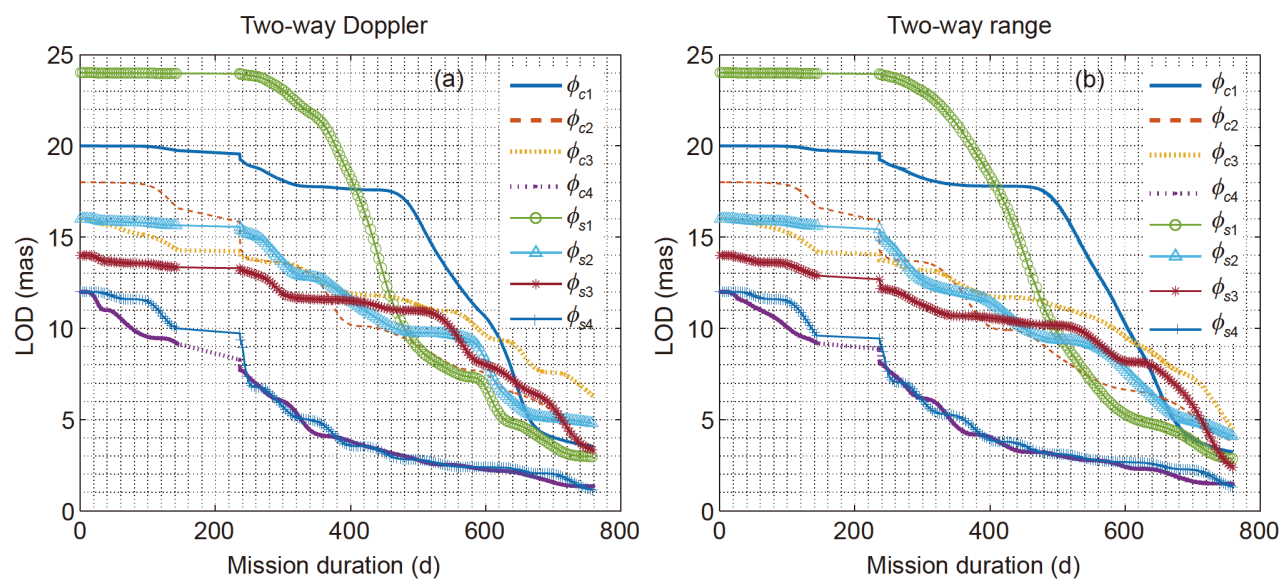

图 6 (网络版彩图)日长变化参数收玫曲线. (a) 使用双程多普勒数据计算日长变化参数的结果; (b) 双程测距数据的计算结果 Figure 6 (Color online) LOD parameters versus tracking duration. (a) The result of using two-way Doppler data to determine LOD parameters; (b) the result of using two-way range data.
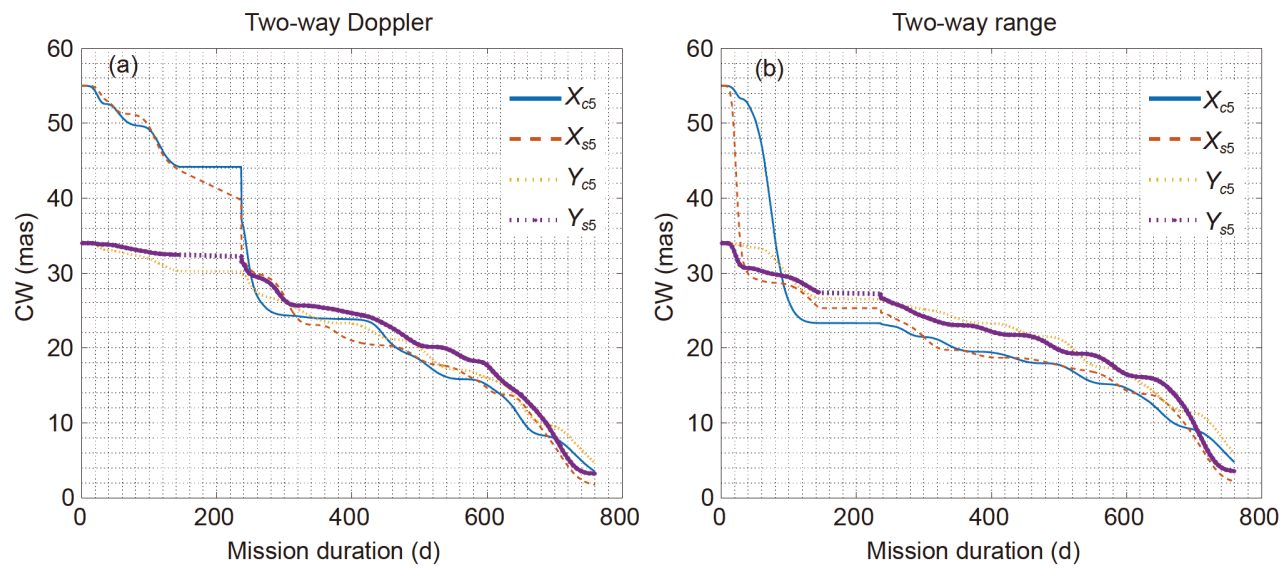

图 7 (网络版彩图)极移参数收玫曲线(只列出了钱德勒摆动的结果). (a) 使用双程多普勒数据计算钱德勒摆动参数的结果; (b) 双程测距数据的计算结果

Figure 7 (Color online) Polar motion parameters versus tracking duration (only Chandler Wobble). (a) The result of using two-way Doppler data to determine the Chandler Wobble; (b) the result of using two-way range data.

\section{6 火星星历误差的影响}

在实际火星定向参数计算过程中，火星星历误差 客观存在，不可避免地会对解算结果产生影响。使用 从1976到2014年收集的火星轨道器和着陆器的跟踪数 据，目前火星预报星历的精度可达几百米 ${ }^{[27]}$. 以往火 星探测器测距跟踪数据的RMS从 $10 \mathrm{~cm}$ 到 $4 \mathrm{~m}$ 不等, 当 存在观测数据时, 火星星历的精度有望达到米级 ${ }^{[13]}$. 为了评估火星星历误差对定向参数解算的影响，重做 上述仿真实验. 鉴于星历误差主要沿轨分布, 在解算 定向参数过程中为火星位置增加一个 $\Delta t$ 的偏移，以此 来模拟火星星历误差. 具有代表性的定向参数解算结
果随星历误差的变化如图9所示.

图9中，横轴表示火星星历误差，纵轴表示定向参 数的真误差与标准差(不确定度)之比, 其比值可反映 解算过程中系统误差的大小. 毫无疑问, 火星星历误 差为定向参数的解算引入了系统误差. 其中, 星历误 差对双程多普勒数据解算定向参数的影响较小, 当火 星星历精度大于 $75 \mathrm{~m}$ 时，定向参数的真误差仍然落在 其三倍标准差的范围之内; 而双程测距数据受星历误 差的影响较大, 只有当火星星历误差小于 $0.15 \mathrm{~m}$ 时, 定 向参数的解算结果才具有可信性. 因此, 使用双程测距 数据计算火星定向参数时, 必须同时解算火星轨道, 以 避免系统误差对定向参数解算带来的影响. 
表 2 着陆器位置和火星定向参数解算精度

Table 2 Accuracy of lander position and MOPs

\begin{tabular}{ccc||ccc}
\hline 符号 & 双程多普勒 $(1 \sigma)$ & 双程测距 $(1 \sigma)$ & 符号 & 双程多普勒 $(1 \sigma)$ & 双程测距 $(1 \sigma)$ \\
\hline$X(\mathrm{~m})$ & 0.35 & 0.43 & $I_{50}, \psi_{50}$ (mas) & $14.47,33.01$ & $9.50,18.42$ \\
$Y(\mathrm{~m})$ & 0.41 & 0.52 & $I_{60}, \psi_{60}(\mathrm{mas})$ & $20.06,43.82$ & $13.39,27.90$ \\
$Z(\mathrm{~m})$ & 5.93 & 0.06 & $I_{70}, \psi_{70}$ (mas) & $2.29,3.51$ & $2.14,2.95$ \\
$\psi_{0}(\mathrm{mas} / \mathrm{yr})$ & 0.75 & 0.35 & $I_{80}, \psi_{80}$ (mas) & $0.49,0.84$ & $0.48,0.83$ \\
$I_{0}(\mathrm{mas} / \mathrm{yr})$ & 0.90 & 0.63 & $I_{90}, \psi_{90}$ (mas) & $0.06,0.17$ & $0.06,0.17$ \\
$\phi_{0}(\mathrm{deg} / \mathrm{d})$ & 0.0000000011 & 0.0000000014 & $X_{c 1}, X_{s 1}$ (mas) & $7.54,5.29$ & $6.55,5.01$ \\
$\phi_{c 1}, \phi_{s 1}(\mathrm{mas})$ & $3.52,2.97$ & $3.22,2.85$ & $X_{c 2}, X_{s 2}$ (mas) & $5.97,6.12$ & $5.49,5.70$ \\
$\phi_{c 2}, \phi_{s 2}(\mathrm{mas})$ & $4.80,4.79$ & $3.82,4.07$ & $X_{c 4}, X_{s 4}$ (mas) & $1.62,1.81$ & $1.62,1.65$ \\
$\phi_{c 3}, \phi_{s 3}(\mathrm{mas})$ & $6.25,3.33$ & $4.48,2.36$ & $X_{c 5}, X_{s 5}$ (mas) & $3.47,1.76$ & $4.74,2.23$ \\
$\phi_{c 4}, \phi_{s 4}(\mathrm{mas})$ & $1.33,1.12$ & $1.48,1.30$ & $Y_{c 1}, Y_{s 1}$ (mas) & $4.80,4.75$ & $4.78,4.75$ \\
$I_{10}, \psi_{10}(\mathrm{mas})$ & $9.01,15.94$ & $7.31,8.46$ & $Y_{c 2}, Y_{s 2}$ (mas) & $6.55,6.73$ & $6.30,6.45$ \\
$I_{20}, \psi_{20}(\mathrm{mas})$ & $4.41,7.45$ & $4.25,7.18$ & $Y_{c 4}, Y_{s 4}$ (mas) & $1.74,1.86$ & $1.74,1.76$ \\
$I_{30}, \psi_{30}(\mathrm{mas})$ & $20.67,45.05$ & $13.23,29.53$ & $Y_{c 5}, Y_{s 5}$ (mas) & $4.63,3.21$ & $5.87,3.55$ \\
$I_{40}, \psi_{40}(\mathrm{mas})$ & $2.97,6.84$ & $2.93,6.60$ & & &
\end{tabular}
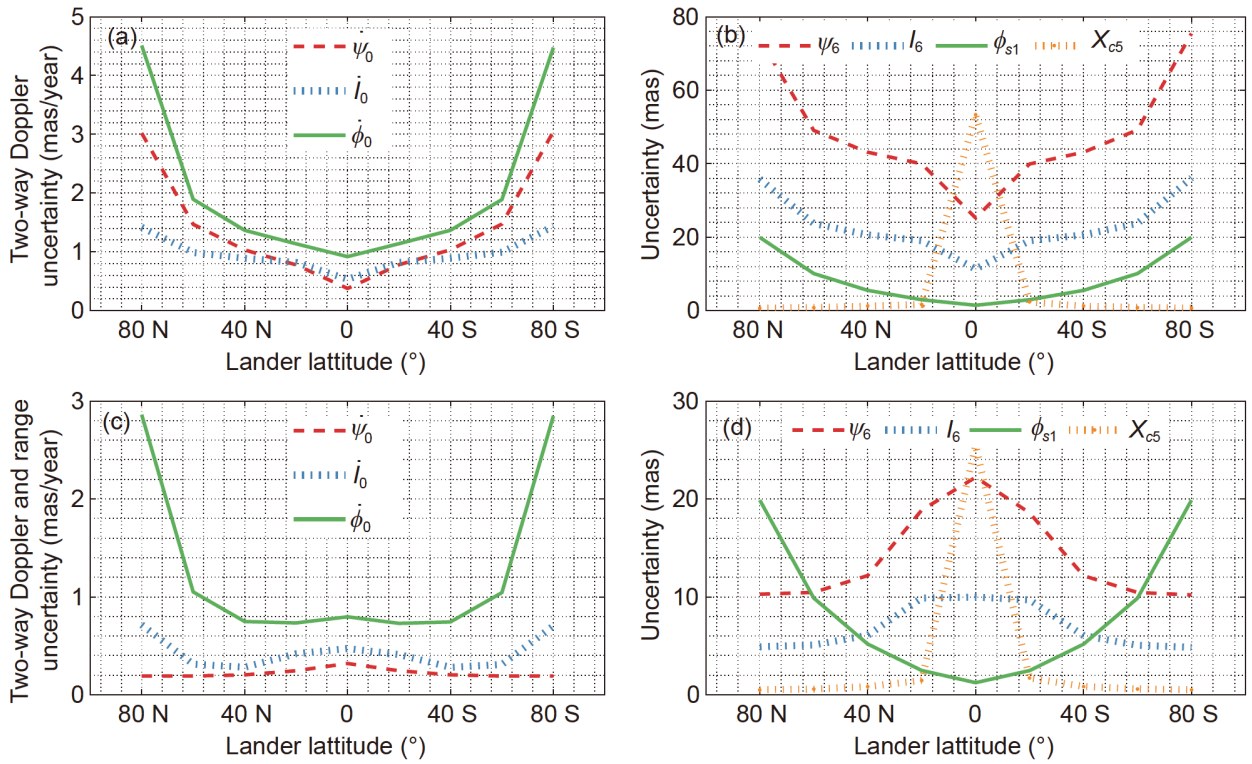

图 8 (网络版彩图)着陆器纬度变化对火星定向参数解算精度的影响(为清晰易读, 只绘制出具有代表性的定向参数). (a), (b) 只使用双程多普勒数据的仿真计算结果; (c), (d) 综合双程多普勒和测距的结果

Figure 8 (Color online) The effect of lander latitude on the accuracy of Mars orientation parameters (for clarity, only representative orientation parameters are drawn). (a), (b) Simulation results using two-way Doppler measurement; (c), (d) combined solution results of two-way Doppler and two-way range.

\section{7 结论}

本文定量仿真分析了着陆器测量进行定位与火星 定向参数解算所能达到的精度. 仿真结果表明, 在当前
测量噪声水平下, 位于北纬 $20^{\circ}$ 的着陆器进行双程多普 勒与双程测距可达到近似的精度水平，几小时跟踪数 据便可使着陆器在赤道面上的精度达到米级; 约 200 任务日的跟踪数据将使岁差参数超越目前的精度，运 

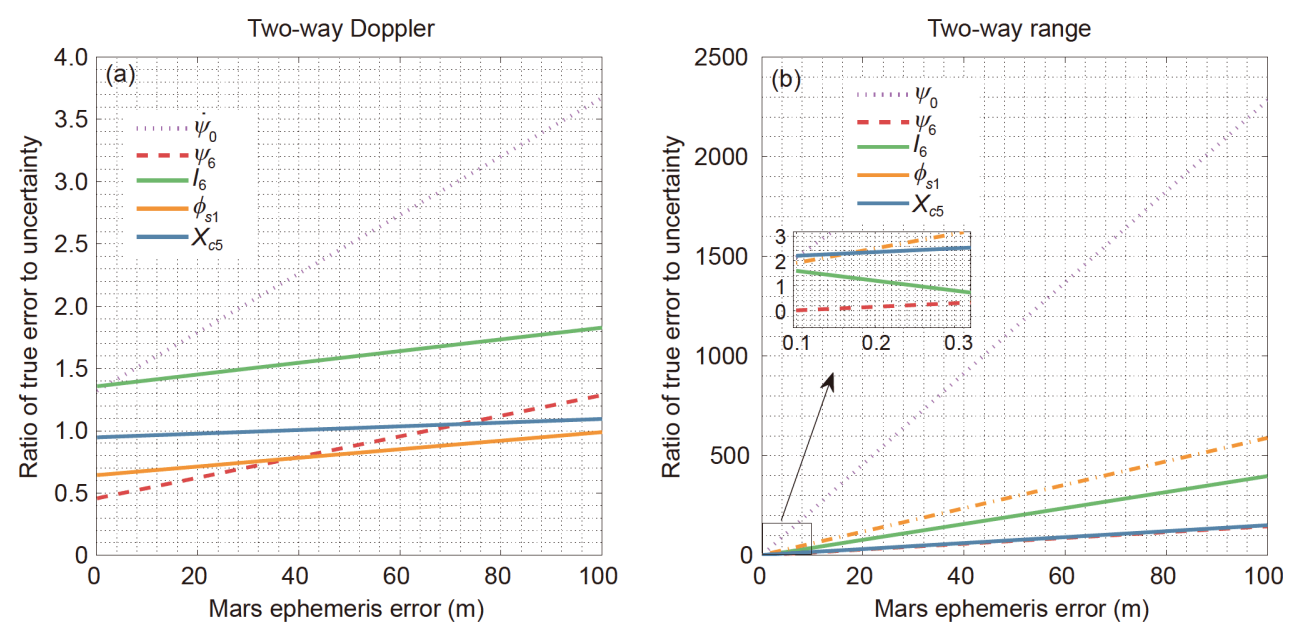

图 9 (网络版彩图) 火星星历误差对火星定向参数解算精度的影响(为清晰易读, 只绘制出具有代表性的定向参数). (a) 使用双 程多普勒数据的情况; (b) 使用双程测距数据的情况

Figure 9 (Color online) The effect of Mars ephemeris error on the accuracy of Mars orientation parameters (for clarity, only representative orientation parameters are drawn). (a) The case of using two-way Doppler data; (b) the case of using two-way range data.

行两年后, 其精度可提高5-10倍. 虽然目前自转速率精 度较高, 但随着任务的持续, 仍可对其解算产生贡献. 章动参数年周期项、半年周期项以及 $1 / 3$ 年周期项可 提高精度至10-30 mas, 而日长变化与钱德勒摆动参数 精度最终可收玫到5-10 mas. 通过仿真分析不同纬度 着陆器的情况, 发现低纬度的着陆器不利于极移参数 的计算, 而高纬度的着陆器不利于自转速率和日长变
化参数的计算，若只进行双程多普勒测量会显著降低 岁差和章动参数的解算精度, 因此对于高纬度的着陆 器来说, 有必要增加距离测量来弥补多普勒测量的不 足. 此外, 分析了火星星历误差对参数解算精度的影响, 显示双程多普勒数据受火星星历误差的影响较小, 而 双程测距数据所受的影响较大, 只有当火星星历误差 小于 $0.15 \mathrm{~m}$ 时, 定向参数的解算结果才具有可信性.

\section{参考文献}

1 Le Maistre S, Rosenblatt P, Rivoldini A, et al. Lander radio science experiment with a direct link between Mars and the Earth. Planet Space Sci, 2012, 68: 105-122

2 Dehant V, Folkner W, Renotte E, et al. Lander radioscience for obtaining the rotation and orientation of Mars. Planet Space Sci, 2009, 57: 1050-1067

3 Dehant V, Le Maistre S, Rivoldini A, et al. Revealing Mars' deep interior: Future geodesy missions using radio links between landers, orbiters, and the Earth. Planet Space Sci, 2011, 59: 1069-1081

4 Yseboodt M, Barriot J P, Dehant V. Analytical modeling of the Doppler tracking between a lander and a Mars orbiter in terms of rotational dynamics. J Geophys Res, 2003, 108: 5076

5 Le Maistre S, Rosenblatt P, Dehant V, et al. Mars rotation determination from a moving rover using Doppler tracking data: What could be done? Planet Space Sci, 2018, 159: 17-27

6 Borderies N, Balmino G, Castel L, et al. Study of Mars dynamics from lander tracking data analysis. Moon Planets, 1980, 22: 191-200

7 Yoder C F, Standish E M. Martian precession and rotation from Viking lander range data. J Geophys Res, 1997, 102: 4065-4080

8 Folkner W M, Yoder C F, Yuan D N, et al. Interior structure and seasonal mass redistribution of Mars from radio tracking of Mars Pathfinder. Science, 1997, 278: 1749-1752

9 Le Maistre S. The rotation of Mars and Phobos from Earth-based Radio-Tracking Observations of a Lander. Dissertation for Doctoral Degree. Brussels: Université Catholique de Louvain, 2013 
10 Kuchynka P, Folkner W M, Konopliv A S, et al. New constraints on Mars rotation determined from radiometric tracking of the Opportunity Mars Exploration Rover. Icarus, 2014, 229: 340-347

11 Folkner W M, Dehant V, Le Maistre S, et al. The rotation and interior structure experiment on the InSight mission to Mars. Space Sci Rev, 2018, 214: 100

12 Reasenberg R D, King R W. The rotation of Mars. J Geophys Res, 1979, 84: 6231-6240

13 Konopliv A S, Yoder C F, Standish E M, et al. A global solution for the Mars static and seasonal gravity, Mars orientation, Phobos and Deimos masses, and Mars ephemeris. Icarus, 2006, 182: 23-50

14 Van Hoolst T, Dehant V, Defraigne P. Chandler wobble and free core nutation for Mars. Planet Space Sci, 2000, 48: 1145-1151

15 Jacobson R A, Konopliv A S, Park R S, et al. The rotational elements of Mars and its satellites. Planet Space Sci, 2018, 152: 107-115

16 Huang Y, Chang S, Li P, et al. Orbit determination of Chang'E-3 and positioning of the lander and the rover. Chin Sci Bull, 2014, 59: 3858-3867

17 Cao J F, Zhang Y, Hu S J, et al. An analysis of precise positioning and accuracy of the CE-3 lunarlander soft landing (in Chinese). Geomatics Inf Sci Wuhan Univ, 2016, 41: 274-278 [曹建峰, 张宇, 胡松杰, 等. 嫦娥三号着陆器精确定位与精度分析. 武汉大学学报(信息科学版), 2016, 41: 274-278]

18 Cao J F, Huang Y, Hu X G, et al. Modeling and application of Doppler data in deep space exploration (in Chinese). J Astron, 2011, 32: 1583-1589 [曹建峰, 黄勇, 胡小工, 等. 深空探测中多普勒的建模与应用. 宇航学报, 2011, 32: 1583-1589]

19 Yang X, Yan J G, Ye M, et al. Development of precise orbit determination software for Mars probe and data processing for MEX (in Chinese). Geomatics Inf Sci Wuhan Univ, 2019, 44: 385-391 [杨轩, 鄢建国, 叶茂, 等. 火星探测器精密定轨软件研制及实测数据处理. 武汉大学学报 (信息科学版), 2019, 44: 385-391]

20 Iess L, Asmar S, Tortora P. MORE: An advanced tracking experiment for the exploration of Mercury with the mission BepiColombo. Acta Astronaut, 2009, 65: 666-675

21 Estefan J A, Thurman S W, Batchelder J A. Earth-based ranging measurements to a Planetary Radio Beacon: An approximate analytic formulation. TDA Progress Report, 1990. 42-103

22 Folkner W M, Kahn R D, Preston R A, et al. Mars dynamics from Earth-based tracking of the Mars Pathfinder lander. J Geophys Res, 1997, 102: 4057-4064

23 Yseboodt M, Dehant V, Péters M J. Signatures of the Martian rotation parameters in the Doppler and range observables. Planet Space Sci, 2017, 144: 74-88, arXiv: 1611.09040

24 Le Maistre S. InSight coordinates determination from direct-to-Earth radio-tracking and Mars topography model. Planet Space Sci, 2016, 121: 1-9 Defraigne P, de Viron O, Dehant V, et al. Mars rotation variations induced by atmosphere and ice caps. J Geophys Res, 2000, 105: 24563-24570 Van den Acker E. Influence of the seasonal winds and the $\mathrm{CO}_{2}$ mass exchange between atmosphere and polar caps on Mars' rotation. J Geophys Res, 2002, 107: 9

27 Folkner W M, Williams J G, Boggs D H, et al. The planetary and lunar ephemerides DE430 and DE431. Interplanet Network Prog Rep, 2014, 196: $1-81$ 


\title{
Mars orientation parameters determination based on direct-to-Earth measurement
}

\author{
YANG Xuan $^{1,2}$, YAN Jian-Guo ${ }^{1 *}$, LE MAISTRE Sebastien ${ }^{2}$, DEHANT Véronique $^{2}$, \\ YE Mao ${ }^{1}$, JIN Wei-Tong ${ }^{1} \&$ LI Fei ${ }^{1}$ \\ ${ }^{1}$ State Key Laboratory of Engineering in Surveying, Mapping and Remote Sensing, Wuhan University, Wuhan 430079, China; \\ ${ }^{2}$ Royal Observatory of Belgium, Brussels B1180, Belgium
}

The Mars orientation parameters (MOPs) are important information for constraining Martian interior properties, ice caps sublimation, and condensation process. Considering the future Mars landing mission, we performed numerical simulations of MOP determination using direct-to-Earth radio signals. The results suggest that after $200 \mathrm{~d}$ of tracking using high-quality levels of both two-way Doppler and two-way range measurements, the MOP uncertainties will be significantly reduced. Then, after about 800 mission days the accuracy of precession parameters will be improved by $5-$ 10 times compared with the present. Also, the accuracy of nutation parameters will reach 10-30 mas, and the accuracy of length-of-day and Chandler wobble parameters will reach 5-10 mas, which meets the need to investigate the internal structure of Mars and the dynamics of the atmosphere. By analyzing the solution results of different latitude landers, twoway range data can provide a good complement to two-way Doppler data. Therefore, it is necessary to perform a twoway range measurement for a high-latitude lander. In addition, the study has also found the limit of the ephemeris error of Mars for MOP determination. When using the two-way Doppler data, the ephemeris error should not be more than $75 \mathrm{~m}$; and for the two-way range measurement, the error should be less than $0.15 \mathrm{~m}$.

Mars lander, Mars rotation parameters, Doppler, range measurement

PACS: $96.30 . \mathrm{Gc}$, 95.55.Pe, $91.10 . \mathrm{Fc}$

doi: 10.1360/SSPMA-2020-0005 